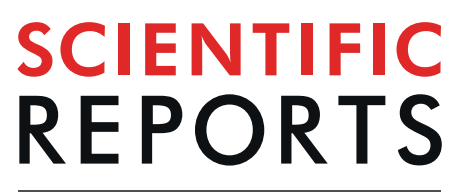

natureresearch

\title{
Mosaic chromosome $Y$ loss is associated with alterations in blood cell counts in UK Biobank men
}

\author{
Shu-Hong Lin ${ }^{1}$, Erikka Loftfield ${ }^{1}$, Josh N. Sampson ${ }^{1}$, Weiyin Zhou ${ }^{1,2}{ }^{2}$, Meredith Yeager ${ }^{1,2}$, \\ Neal D. Freedman ${ }^{1}$, Stephen J. Chanock ${ }^{1}$ \& Mitchell J. Machiela ${ }^{1 *}$ \\ Mosaic loss of $Y$ chromosome ( $\mathrm{mLOY}$ ) is the most frequently detected somatic copy number alteration \\ in leukocytes of men. In this study, we investigate blood cell counts as a potential mechanism linking \\ $\mathrm{mLOY}$ to disease risk in 206,353 UK males. Associations between mLOY, detected by genotyping \\ arrays, and blood cell counts were assessed by multivariable linear models adjusted for relevant risk \\ factors. Among the participants, mLOY was detected in 39,809 men. We observed associations between \\ $\mathrm{mLOY}$ and reduced erythrocyte count $\left(-0.009[-0.014,-0.005] \times 10^{12}\right.$ cells $\left./ \mathrm{L}, p=2.75 \times 10^{-5}\right)$ and \\ elevated thrombocyte count $\left(5.523[4.862,6.183] \times 10^{9}\right.$ cells $\left./ L, p=2.32 \times 10^{-60}\right)$ and leukocyte count \\ $\left(0.218[0.198,0.239] \times 10^{9}\right.$ cells $\left./ \mathrm{L}, p=9.22 \times 10^{-95}\right)$, particularly for neutrophil count $(0.174 \times[0.158$, \\ $0.190] 10^{9}$ cells $\left./ \mathrm{L}, p=1.24 \times 10^{-99}\right)$ and monocyte count $(0.021$ [0.018 to 0.024$] \times 10^{9}$ cells $/ \mathrm{L}$, \\ $\left.p=6.93 \times 10^{-57}\right)$, but lymphocyte count was less consistent $\left(0.016[0.007,0.025] \times 10^{9}\right.$ cells $/ \mathrm{L}$, \\ $\left.p=8.52 \times 10^{-4}\right)$. Stratified analyses indicate these associations are independent of the effects of aging \\ and smoking. Our findings provide population-based evidence for associations between $\mathrm{mLOY}$ and \\ blood cell counts that should stimulate investigation of the underlying biological mechanisms linking \\ $\mathrm{mLOY}$ to cancer and chronic disease risk.
}

Recently, large molecular epidemiology studies have shown that hematopoietic cells can undergo postzygotic mutations resulting in somatic copy number alterations, which can give rise to daughter cells with the same genomic aberration. Clonal expansion of cells bearing a somatic mutation results in clonal mosaicism ${ }^{1}$. Clonal mosaicism can be driven by somatic mutations affecting genes frequently mutated in myelodysplastic disease (referred to as clonal hematopoiesis of indeterminate potential (CHIP)) $)^{2}$ or be the result of acquired copy number aberrations. The most frequently detected somatic copy number alteration in circulating leukocytes is mosaic loss of the $\mathrm{Y}$ chromosome (mLOY) in males ${ }^{3-5}$. The prevalence of $\mathrm{mLOY}$ is age-related, increasing substantially after age 50 in men. Likewise, CHIP is common in the elderly, with recent evidence suggesting CHIP and mLOY may co-occur ${ }^{6}$. Exposure to cigarette smoking ${ }^{4,7}$ has also been well established as a risk factor for mLOY and recently, early evidence suggests that air pollution could also be associated with $\mathrm{mLOY}^{8}$. Epidemiologic studies have uncovered potential associations between $\mathrm{mLOY}$ and increased risk of $\operatorname{cancer}^{3-5}$, neurodegenerative diseases ${ }^{9}$, and cardiovascular diseases ${ }^{10,11}$. Similarly, CHIP has been associated with select cancers and cardiovascular disease $^{2}$. Although common germline variants near important cell-cycle regulation and cancer susceptibility genes have been identified through genome-wide association studies of $\mathrm{mLOY}^{4,12}$, the underlying biologic mechanisms linking mLOY to chronic disease risk are likely complex.

We investigated possible associations between mLOY and clinical measures, here the composition of the classical hematologic compartments in the UK Biobank, which has available genomic and blood cell count data on over 220,000 men, providing a large well-characterized population to investigate these questions. We performed multivariable, stratified and mediation analyses to evaluate the association of mLOY with blood cell count and distribution. Our study provides a unique, population-based investigation of changes in blood cell counts associated with a somatic mutation. Our findings indicate robust associations between mLOY and leukocyte, erythrocyte and thrombocyte counts, independent of age and cigarette smoking.

${ }^{1}$ Division of Cancer Epidemiology and Genetics, National Cancer Institute, Rockville, 9609 Medical Center Drive MSC 9776, Bethesda, Maryland, 20892, USA. ${ }^{2}$ Cancer Genomics Research Laboratory, Frederick National Laboratory for Cancer Research, Frederick, Maryland, 8717 Grovemont Circle, Gaithersburg, MD, 20877, USA. *email: mitchell. machiela@nih.gov 


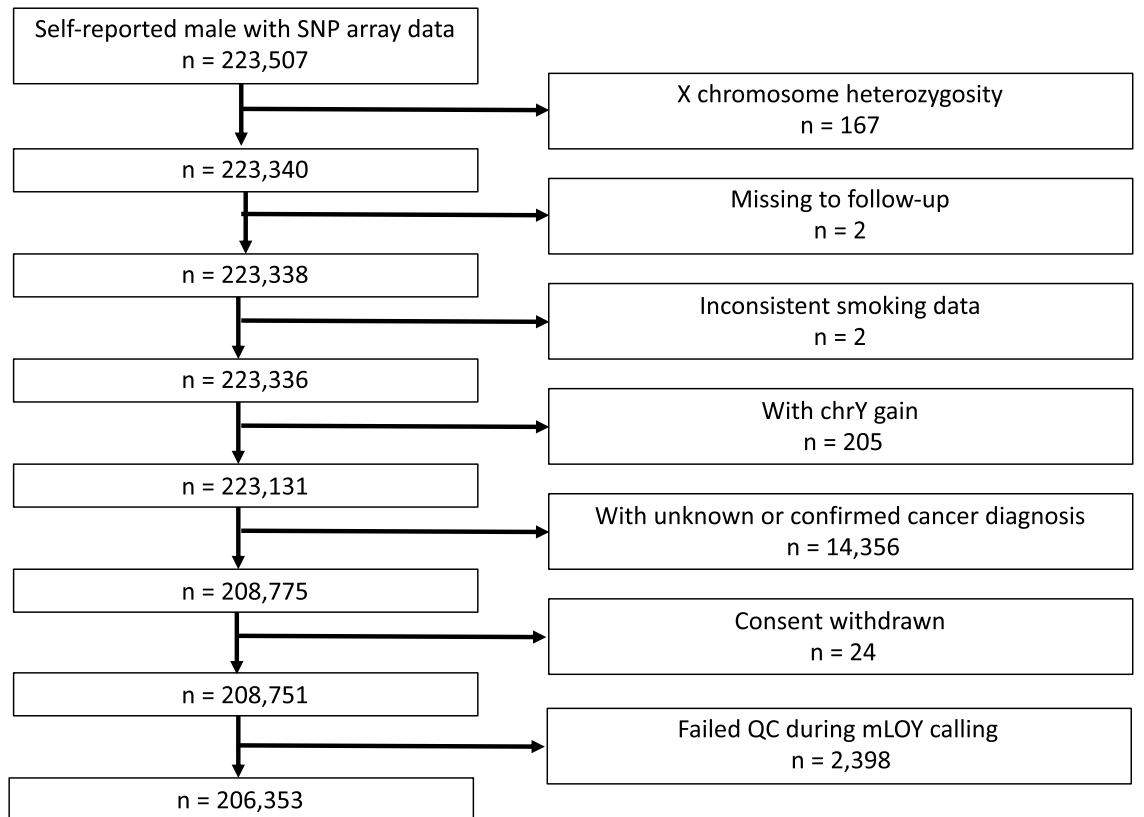

Figure 1. Number of participants. The number of participants excluded by each criterion was shown.

\section{Methods}

The current analyses were extension of our previous studies ${ }^{5,13,14}$ which included population-based data from 223,336 males between age 37 and 73 recruited between 2007 to 2010 from the UK Biobank ${ }^{15,16}$. After providing informed consent, each participant provided a blood sample, answered a detailed health and lifestyle questionnaire, and had physical measurements taken. Collected blood was held at $4{ }^{\circ} \mathrm{C}$ and sent to a central processing laboratory in temperature-controlled boxes. Samples were processed, aliquoted and cryopreserved at $-80^{\circ} \mathrm{C}$ or $-196^{\circ} \mathrm{C}$. One tube of blood was loaded on a Beckman Coulter LH750 to provide direct measurement of various blood cell counts and indices including but not limiting to hematocrit, plateletcrit, mean corpuscular volume, hemoglobin, mean corpuscular hemoglobin, mean corpuscular hemoglobin concentration, mean sphered cell volume, mean platelet volume, platelet distribution width, immature reticulocyte fraction, and high light scatter reticulocyte proportion.

Blood-derived DNA from UK Biobank men was extracted starting early 2013 and genotyped on Affymetrix UK BiLEVE or UK Biobank Axiom arrays. mLOY was measured by two different methods. The first measure of mLOY was made by examining the median $\log R$ ratio (mLRR) of 691 single nucleotide polymorphisms spanning the male specific region of the Y chromosome. LRR is a measure of probe signal intensity on the genotyping array with negative values across contiguous variants indicating evidence for a copy number loss and positive values indicating copy number gain. Men with $\mathrm{mLRR}>0.15$ could possibly have a mosaic gain of $\mathrm{Y}$ chromosome or a constitutional extra copy of the Y chromosome (XYY syndrome) and were removed (205 men, 0.09\%). We also apply the methods proposed by Thompson et al. ${ }^{14}$ to call dichotomized mLOY. For clarity, we use the term mLOY for dichotomized mLOY calls by Thompson et al.

Our final analytical set included 206,353 self-reported men who reported no prior cancer history at recruitment $(\mathrm{n}=14,356$ excluded), did not have $X$ chromosome heterozygosity $(\mathrm{n}=167$ excluded), had consistent smoking data $(n=2$ excluded), were without evidence of copy number gains of $Y$ chromosome $(n=205$ excluded) and passed quality control during the dichotomized mLOY detection step $(\mathrm{n}=2,394$ excluded) as shown in Fig. 1. Multivariable linear regression was employed to identify associations between lifestyle factors, mLOY and blood cell counts. Factors which might influence blood cell counts (smoking ${ }^{17}, \mathrm{BMI}^{18}$, alcohol consumption $^{19}$, diabetes ${ }^{20}$, hypertension ${ }^{21}$, and hypoercholesterolemia ${ }^{22}$ ) were included in multivariable models. For interaction between smoking and mLOY, we fit models with mLRR, 3-level smoking status (never, former, current), interaction terms between mLRR and smoking, and all other aforementioned covariates. We adjusted for continuous age, age squared ${ }^{13}$, race/ethnicity, smoking, alcohol consumption, continuous body mass index (BMI), self-reported diabetes, hypertension, and hypercholesterolemia. When modeling mLRR, we applied the following formula to calculate standardized mLRR for easier interpretation of the regression coefficient. We first subtracted mean of raw mLRR $\left(m_{L R R}\right)$ from all raw mLRR, divided the difference by standard deviation (S.D.) of $\mathrm{mLRR}_{\mathrm{r}}$, and flipped the sign (to have effect estimates in the same direction as the dichotomous mLOY estimates).

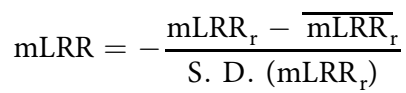

A 25-level smoking status variable was created according to prior literature ${ }^{13}$ and used in the majority of our models. In brief, a detailed smoking history variable was created by combining information on baseline smoking 
status, smoking intensity, time since quitting, and type of tobacco product smoked (i.e., cigarettes or cigars/pipes). Indicator variables were created for each category and "never smokers" were used as the reference group. After accounting for skip patterns, a small percentage of respondents were missing one or more pieces of information. Because we had some, but not complete, smoking information on these respondents we created a number of indicator variables for each of the partial missing categories. Alcohol consumption was coded in 7 levels (never, former, occasional, 1-3 drink/month, 1-2 drink/week, 3-4 drink/week) according to the history and frequency of consumption. Continuous BMI was used in most models while a 5-level BMI defined by the World Health Organization (WHO) was also presented for reference. All missing data in categorical variables were coded as 'missing' and included in the analysis. A significance level was set at 0.001 to account for multiple comparisons $(0.05 / 50$ independent tests for all indicator variables). To evaluate the effect of any potential residual confounding from smoking and age in the reported association between mLOY and blood cell counts, we conducted a sensitivity analysis restricted to strata of ever or never smokers and individuals older or younger than 65 years old. We chose 65 years old because the prevalence of mLOY increased exponentially between 60 to 70 years old even for never smokers (Fig. S1).

A polygenic risk score (PRS) was created to estimate the effect of genetic contributors to mLOY. The PRS was calculated by scoring risk alleles in 156 variants previously found to be associated with mLOY risk using the formula:

$$
\operatorname{PRS}_{i}=\sum_{j=1}^{156} \beta_{i}\left(\operatorname{SNP}_{i j}\right)
$$

$i$ denotes an individual while $j$ denotes a SNP being scored. The number of risk alleles of each variant $\left(\mathrm{SNP}_{i j}\right)$ were weighted by reported estimates for log odds ratio of risk alleles $(\beta)^{23}$. Then, blood cell counts and other indices were regressed against the PRS instrumental variable to investigate for potential associations between mLOY PRS and blood counts. Mediation analyses were conducted to estimate the direct and indirect effect among smoking, mLOY, and blood cell counts while controlling for BMI, ethnicity, alcohol consumption, diabetes, hypertension and hypercholesterolemia using the "mediation" package in $\mathrm{R}^{24}$.

Finally, we performed reciprocal Mendelian randomization (MR) on previously reported mLOY-associated SNPs as well as blood cell count-associated SNPs obtained from PhenoScanner ${ }^{25,26}$. We queried for respective blood cell counts in PhenoScanner and discarded multi-allelic SNPs. The final list of SNPs included 270 for leukocyte count ${ }^{27-39}, 1,888$ for erythrocyte count ${ }^{29,31-36,39-48}$, and 662 for thrombocyte count ${ }^{29,31-36,44,49-55}$. Estimation of causal association was carried out by the "MendelianRandomization" package ${ }^{56}$. All statistical analyses were performed in R version 3.5.2 and plots were created using the "ggplot2" 57 and "sjPlot"58 packages.

Ethical approval. The UK Biobank received ethical approval from the research ethics committee (REC reference for UK Biobank 21552) and all participants provided signed informed consent at enrollment and all research was performed in accordance with relevant guidelines/regulations. All data used in this analysis is available through application to the UK Biobank.

\section{Results}

Patient characteristics are shown in Table 1 based on their mLOY status. Among the 206,353 UK Biobank male subjects in our analytic set, we detected mLOY in 39,809 men (19.29\%). Compared to participants without mLOY, those with mLOY were older $\left(6.683[6.598,6.768]\right.$ years, $\left.p<5 \times 10^{-324}\right)$, more likely to be white $\left(\mathrm{OR}=2.822[2.560,3.118], 4.014[3.464,4.682]\right.$ compared to Asian and Black, respectively. $p<5 \times 10^{-324}$ for both), more likely to smoke $(\mathrm{OR}=1.588[1.551,1.627]$ and $1.973[1.910,2.038]$ for former and current compared to never smokers, respectively. $p<5 \times 10^{-324}$ for both), more likely to consume alcohol $(\mathrm{OR}=1.451[1.324,1.591]$ and $1.326[1.234,1.426]$ for former and current compared to never drinkers, $p=8.88 \times 10^{-16}$ and $4.00 \times 10^{-15}$, respectively), less likely to have $\mathrm{BMI}>35\left(\mathrm{OR}=0.645[0.513,0.820], p<5 \times 10^{-324}\right)$, more likely to have diabetes $\left(\mathrm{OR}=1.121[1.072,1.171], p=6.71 \times 10^{-7}\right)$, hypertension $\left(\mathrm{OR}=1.344[1.314,1.376], p<5 \times 10^{-324}\right)$, and hypercholesterolemia $\left.(\mathrm{OR}=1.509[1.468,1.552]], p<5 \times 10^{-324}\right)$. As expected, as individuals age, the prevalence of $\mathrm{mLOY}$ increased exponentially (Fig. S1). There was no significant difference for mLOY prevalence by assessment center and region (Table S1). In univariable analyses, we detected associations $(\mathrm{p}<0.001)$ between mLOY and counts of six blood cell populations including leukocytes, erythrocytes, thrombocytes, lymphocytes, monocytes, and neutrophils (Fig. 2). We examined possible confounding by immune-related diseases identified in inpatient records and did not identify any statistically significant association between these diseases and mLOY (Table S2).

Multivariable analyses further confirmed this relationship between mLOY and blood cell count for erythrocytes $\left(\mathrm{OR}_{\mathrm{mLOY}}=-0.009[-0.014,-0.005] \times 10^{12} \mathrm{cells} / \mathrm{L}, p_{\mathrm{mLOY}}=2.75 \times 10^{-5} ; \beta_{\mathrm{mLRR}}=-0.009[-0.010\right.$, $-0.007] \times 10^{12}$ cells $/ \mathrm{L}, p_{\mathrm{mLRR}}=8.73 \times 10^{-23}$ for categorical $\mathrm{mLOY}$ and increase in each standard deviation of mLRR, respectively with all following estimates, $95 \%$ CIs, and $\mathrm{p}$ values reported in the same order), leukocytes $\left(\mathrm{OR}_{\mathrm{mLOY}}=0.218[0.198,0.239] \times 10^{9}\right.$ cells $/ \mathrm{L}, p_{\mathrm{mLOY}}=9.22 \times 10^{-95} ; \beta_{\mathrm{mLRR}}=0.058[0.050$ to 0.066$] \times 10^{9}$ cells $/ \mathrm{L}$, $\left.p_{\mathrm{mLRR}}=6.48 \times 10^{-45}\right)$ and thrombocytes $\left(\mathrm{OR}_{\mathrm{mLOY}}=5.523[4.862,6.183] \times 10^{9}\right.$ cells $/ \mathrm{L}, p_{\mathrm{mLOY}}=2.32 \times 10^{-60}$; $\beta_{\mathrm{mLRR}}=2.321[2.063,2.579] \times 10^{9}$ cells $\left./ \mathrm{L}, p=2.41 \times 10^{-69}\right)($ Fig. 3; Table S3). Among these blood cell count associations, $\mathrm{mLOY}$ was positively associated in all cases except for erythrocyte count where an inverse association was observed. For leukocyte populations, we observed elevated monocyte $\left(\mathrm{OR}_{\mathrm{mLOY}}=0.021[0.018,0.024] \times 10^{9}\right.$ cells $/ \mathrm{L}, p_{\mathrm{mLOY}}=6.93 \times 10^{-57} ; \beta_{\mathrm{mLRR}}=0.005[0.004,0.006] \times 10^{9}$ cells $\left./ \mathrm{L}, p_{\mathrm{mLRR}}=5.24 \times 10^{-25}\right)$ and neutrophil $\left(\mathrm{OR}_{\mathrm{mLOY}}=0.174[0.158,0.190] \times 10^{9} \mathrm{cells} / \mathrm{L}, p_{\mathrm{mLOY}}=1.24 \times 10^{-99} ; \beta_{\mathrm{mLRR}}=0.055[0.048,0.061] \times 10^{9} \mathrm{cells} / \mathrm{L}\right.$, $p_{\mathrm{mLRR}}=4.81 \times 10^{-65}$ ) counts associated with mLOY (Table S4). We found inconsistent evidence for an overall association between $\mathrm{mLOY}$ and the overall lymphocyte count $\left(\mathrm{OR}_{\mathrm{mLOY}}=0.016[0.007,0.025] \times 10^{9} \mathrm{cells} / \mathrm{L}\right.$, $p_{\mathrm{mLOY}}=8.52 \times 10^{-4} ; \beta_{\mathrm{mLRR}}=-0.002[-0.005,0.002] \times 10^{9}$ cells $\left./ \mathrm{L}, p_{\mathrm{mLRR}}=0.345\right)$. While certain immune-related 


\begin{tabular}{|c|c|c|c|}
\hline Characteristics & Normal & mLOY & $P^{a}$ \\
\hline Participants & $166548(80.7)$ & $39809(19.3)$ & NA \\
\hline Age (mean,SD) & $55.199(8.168)$ & $61.882(5.784)$ & $<5 \times 10^{-324}$ \\
\hline \multicolumn{4}{|l|}{ Ethnicity } \\
\hline White & $155443(80)$ & $38790(20)$ & Ref \\
\hline Mixed & $922(90.5)$ & $97(9.5)$ & $<5 \times 10^{-324}$ \\
\hline Asian & $4932(91.9)$ & $436(8.1)$ & $<5 \times 10^{-324}$ \\
\hline Black & $2914(94.2)$ & $181(5.8)$ & $<5 \times 10^{-324}$ \\
\hline Other & $1648(92.3)$ & $138(7.7)$ & $<5 \times 10^{-324}$ \\
\hline \multicolumn{4}{|l|}{ Smoking status } \\
\hline Never & $86007(84.8)$ & $15474(15.2)$ & Ref \\
\hline Former & $60851(77.8)$ & $17388(22.2)$ & $<5 \times 10^{-324}$ \\
\hline Current & $19072(73.8)$ & $6770(26.2)$ & $<5 \times 10^{-324}$ \\
\hline \multicolumn{4}{|l|}{ Alcohol drinking } \\
\hline Never & $4877(84.7)$ & $883(15.3)$ & Ref \\
\hline Former & $5660(79.2)$ & $1487(20.8)$ & $8.88 \times 10^{-16}$ \\
\hline Current & $155837(80.6)$ & $37408(19.4)$ & $4.00 \times 10^{-15}$ \\
\hline \multicolumn{4}{|l|}{ Body mass index } \\
\hline $18.5-$ & $339(78.3)$ & $94(21.7)$ & 0.279 \\
\hline 18.5 to 25 & $39733(80.4)$ & $9702(19.6)$ & Ref \\
\hline 25 to 30 & $80774(80.3)$ & $19853(19.7)$ & 0.636 \\
\hline 30to35 & $32845(81.4)$ & 7507 (18.6) & $1.08 \times 10^{-4}$ \\
\hline $35+$ & $9864(84.8)$ & $1767(15.2)$ & $<5 \times 10^{-324}$ \\
\hline \multicolumn{4}{|l|}{ Diseases } \\
\hline Diabetes & $9870(79)$ & $2625(21)$ & $6.71 \times 10^{-7}$ \\
\hline Hypertension & 48007 (77.4) & $14033(22.6)$ & $<5 \times 10^{-324}$ \\
\hline Hypercholesterolemia & $24396(74.9)$ & $8192(25.1)$ & $<5 \times 10^{-324}$ \\
\hline
\end{tabular}

Table 1. Patient characteristics by mLOY status. Abbreviations: mLOY: mosaic loss of the Y chromosome. aFisher's exact test with mid-p method.

diseases were associated with blood cell counts (Tables S5 and S6), these diseases occurred in less than 1\% of individuals and inclusion of these diseases did not significantly alter the estimates for mLOY and other risk factors. All observed blood count associations demonstrated a dose-response relationship which is evident in the mLRR results. The most prominent association was observed for neutrophil count in which men with mLOY had $1.74 \times 10^{8}$ more cells per liter compared to men without detectable mLOY. This is equivalent to an average increase of $4.153 \%$ in median neutrophil count among men without mLOY. As a comparison, among erythrocytes, the least associated blood cells, men with mLOY had $9 \times 10^{9}$ fewer erythrocyte per liter compared to men without $\mathrm{mLOY}$, a decrease equivalent to $0.189 \%$ of the mean erythrocyte count in individuals without detectable mLOY.

In addition to mLOY, the multivariable model also provided estimates for other potential factors associated with blood cell counts. Smokers who smoke $>40$ cigarettes per day had leukocyte count $36.91 \%$ higher than the median leukocyte count of never smokers with the elevation being evenly distributed across lymphocytes, monocytes, and neutrophils (Figs. 3 and S2). Black ethnicity was associated with 19.26\% less leukocyte and $10.61 \%$ less thrombocyte compared to Whites, and the decrease in leukocyte count was more prominent among monocytes (19.80\%) and neutrophils (32.67\%), but relatively mild in lymphocytes (8.56\%) suggesting a preferential activation of innate immunity. Alcohol consumption, even among daily drinkers, is mildly associated with blood cell counts, and the largest effect size was observed for decrease in leukocyte count (4.14\%) when compared to never drinkers. Another factor associated with blood cell counts is body mass index (BMI) where having BMI greater than 35 was associated with increased leukocyte (13.60\%), erythrocyte (2.92\%), lymphocyte (16.61\%), monocyte $(16.86 \%)$, and neutrophil (11.72\%) counts as well as decreased thrombocyte count (1.91\%) compared to those with normal BMI (18.5 to 25).

As indicated in Table S7, neutrophil-lymphocyte ratio (NLR), a measure used to evaluate systemic inflammation in cancer patients, was found to be significantly elevated in men with $\mathrm{mLOY}\left(\mathrm{OR}_{\mathrm{mLOY}}=0.061[0.045\right.$, $\left.0.077], p_{\mathrm{mLOY}}=2.98 \times 10^{-14}\right)$. The thrombocyte-lymphocyte ratio (TLR), a prognostic indicator for select cancer types $^{59-62}$, was also found to be elevated in men with $\mathrm{mLOY}\left(\mathrm{OR}_{\mathrm{mLOY}}=1.288[0.564,2.012], p_{\mathrm{mLOY}}=4.90 \times 10^{-4}\right)$. These mLOY associations with NLR and TLR showed robust dose response relationships and demonstrated significant trends with continuous $\operatorname{mLRR}\left(p_{\mathrm{mLR}}=1.39 \times 10^{-33}\right.$ and $3.31 \times 10^{-32}$, respectively), suggesting robust relationships for mLOY with measures of both systematic inflammation and cancer prognosis.

To better understand the changes in erythrocyte and platelet counts, we also explored associations between mLOY and multiple erythrocyte indices. Both univariable (Fig. 2) and multivariable (Tables S8 and S9) regression on erythrocyte-related indices indicated that mLOY was associated with an increase in mean corpuscular 

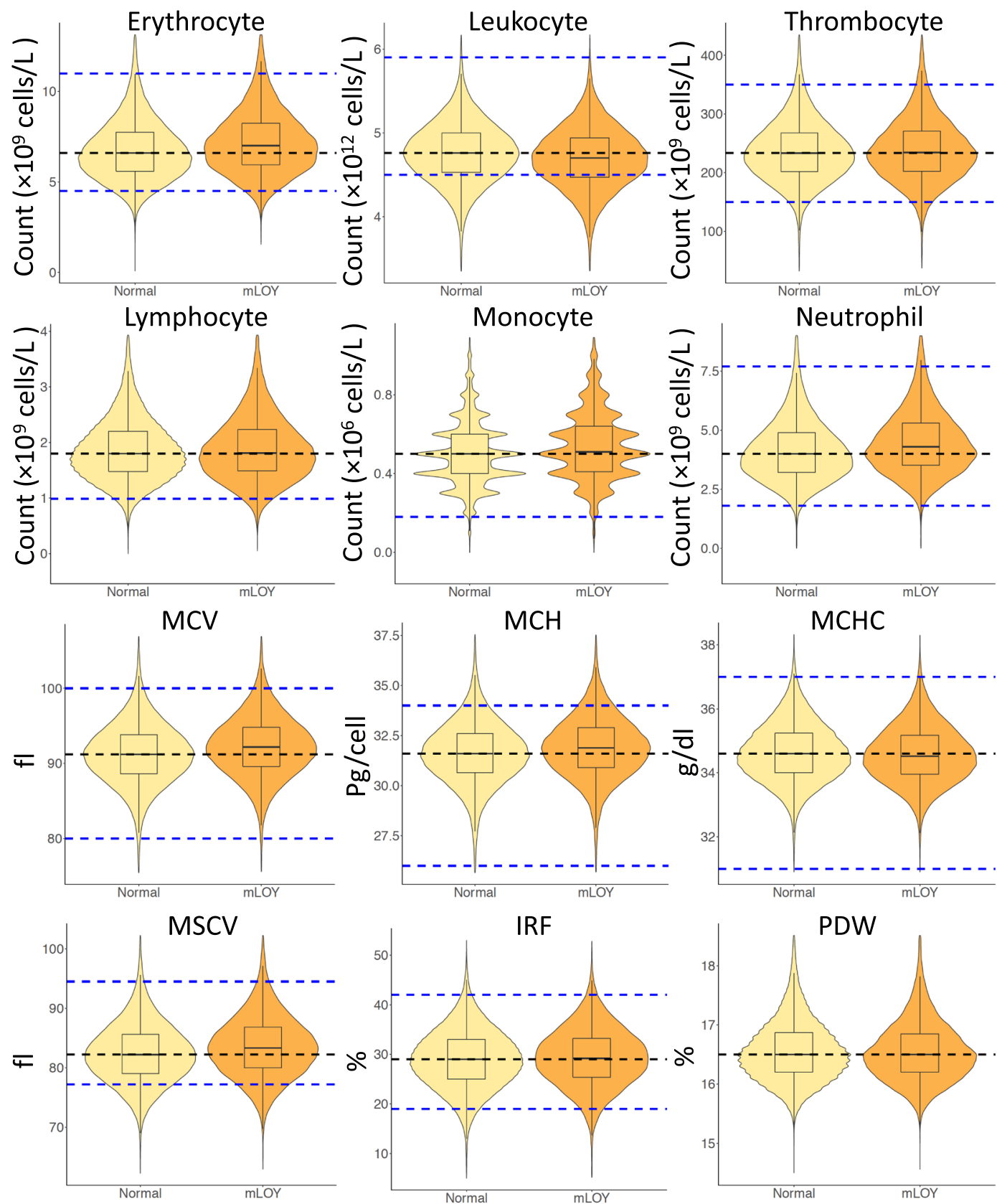

Figure 2. Blood cell counts and indices by mLOY status. Various blood cell counts among men with different mLOY status were observed in univariate analyses. Yellow, Normal: men with normal chromosome Y; Orange, mLOY: men with mLOY. All displayed counts and indices had $p<0.001$ when comparing between participants with or without mLOY. Black dashed lines: median count among normal individuals. Blue dashed lines: reference ranges from prior studies ${ }^{73-75}$. MCV: mean corpuscular volume. $\mathrm{MCH}$ : mean corpuscular hemoglobin. MCHC: mean corpuscular hemoglobin concentration. MSCV: mean sphered corpuscular volume. IRF: immature reticulocyte fraction. PDW: platelet distribution width.

hemoglobin $(\mathrm{MCH})$, mean corpuscular volume $(\mathrm{MCV})$, immature reticulocyte fraction (IRF), mean sphered cell volume (MSCV), and high light scatter reticulocyte percentage (HLSRP) as well as decrease in hemoglobin (Hb).

To evaluate the potential effect of possible residual confounding from smoking, we conducted a sensitivity analysis restricted to strata of ever or never smokers. For leukocyte, erythrocyte, thrombocyte, monocyte, and neutrophil count, associations with $\mathrm{mLOY}$ and cell count remained significant, but the effect size was larger in ever smokers for all counts except thrombocyte count. (Tables S10-S13). Interestingly, lymphocyte count was only significantly increased in ever smokers. We further tested for interactions between mLOY and smoking on changes in blood cell count. mLRR was found to significantly interact with current smoking status in analyses of leukocyte $\left(p=5.06 \times 10^{-31}\right)$, thrombocyte $\left(p=4.18 \times 10^{-6}\right)$, lymphocyte $\left(p=5.25 \times 10^{-11}\right)$, and neutrophil $\left(p=2.26 \times 10^{-25}\right)$ count. Likewise, significant interactions between mLRR and former smoking status were also 


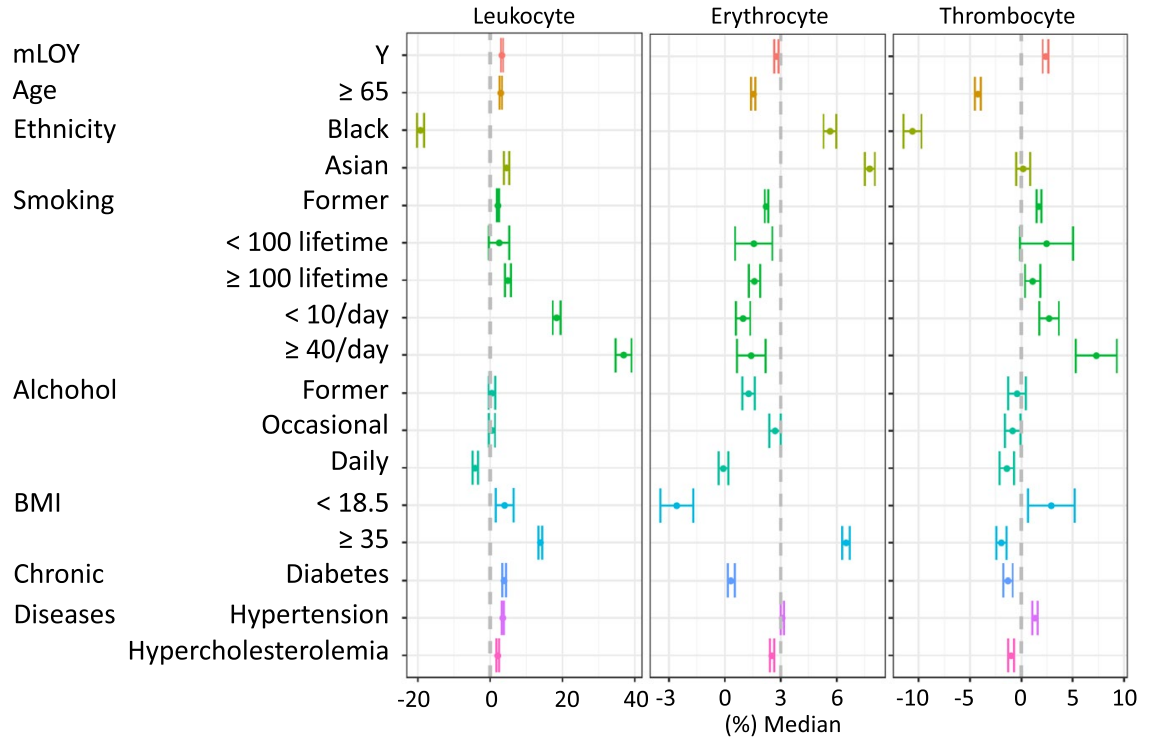

Figure 3. Relative impact of selected risk factors associated with leukocyte, erythrocyte, and thrombocyte counts. Multivariable linear regression models adjusted for age, age squared, race/ethnicity, smoking, alcohol consumption, body mass index (continuous variable), diabetes, hypertension, and hypercholesterolemia. The reference group for categorical variables were no mLOY, Caucasian, never smoker, never drinker, $18.5 \leq$ body mass index $<25$, no diabetes, no hypertension, no hypercholesterolemia. The point estimates, confidence interval, and $p$-values can be found in Table S1.

seen for leukocyte $\left(p=5.30 \times 10^{-8}\right)$ and neutrophil $\left(p=2.33 \times 10^{-6}\right)$ count. Inclusion of interaction terms modified the magnitude of association between mLRR and blood cell counts, but the direction of association between mLRR and cell counts remained the same except for lymphocyte count where the direction of association flipped in never smokers (Fig. S3). The associations between mLOY and NLR remained statically significant in both ever and never smokers (Table S14). We observed a stronger association between TLR and mLOY in never smokers (Table S15).

When stratified by age $(<65$ and $\geq 65$ ), all blood cell counts remained significantly associated with mLOY at similar magnitude except for lymphocyte counts which were only marginally significant in the $\geq 65$-year-old group (Tables S16-S19). As for NLR and TLR the magnitude of association between TLR and mLRR more than doubled in the $\geq 65$ age group (Tables S20 and S21).

We also constructed a polygenic risk score using 156 SNPs previously found to be associated with $\mathrm{mLOY}^{23}$ as an instrumental variable to further investigate the association between mLOY and blood cell counts. As shown in Table S22, the mLOY PRS was significantly associated with leukocyte, erythrocyte, thrombocyte, monocyte, and neutrophil counts and the effect sizes closely mirrored those seen observationally for mLRR. Interestingly, the effect estimate for erythrocyte count was in the inverse direction for the mLOY PRS, suggesting potential non-genetic influences such as malnutrition or hypothyroidism could modify this association. The association between the mLOY PRS and blood cell counts was also examined in UK Biobank females and observed similar results as in males. We did not observe an association between mLOY PRS and overall lymphocyte count in the UK Biobank (Table S22).

In addition, we conducted reciprocal MR with SNPs associated with mLOY and blood cell counts (Table S23 and Fig. S4). We noted bi-directional effects for leukocyte count and thrombocyte count suggesting a potential shared biological process between mLOY and blood cell counts, although further studies are needed to confirm these findings. For erythrocyte counts, a potential effect was only observed from erythrocyte to mLOY, although the estimated effect size was small suggesting environmental or non-genetic contributors may also be important in this relationship.

Mediation analyses were also conducted to investigate whether mLOY acts as a potential mediator of the association between age or smoking and blood cell count. We found evidence suggesting most associations between age and blood cell counts were only partially mediated by mLOY (Tables S24 and S25). The estimated proportion of effect mediated by mLOY was less than $4 \%$, suggesting any potential mediation effect of mLOY on age-related changes in blood cell counts only accounts for a small proportion of the total effect. We also found limited evidence suggesting mLOY mediated the association between smoking and blood cell counts (Tables S26 and S27) including leukocytes, erythrocytes, thrombocytes, monocytes and neutrophils with estimated proportion mediated of less than $2 \%$ of the total effect. 


\section{Discussion}

We present evidence from a large cross-sectional study that demonstrates significant population-based associations between men with mosaic loss of the Y chromosome (mLOY) and circulating blood counts, as measured in a complete blood count. While differences observed remain within the range of expected healthy counts, some men with high proportions of mLOY had substantial deviations in blood cell counts, which could be a harbinger of chronic disease risk.

In a 2018 report by Loh et al., enrichment of autosomal mosaic events was observed among people with abnormal blood cell indices in UK Biobank ${ }^{63}$. For instance, the odds of having a copy number neutral mosaic event in chromosome 9p was $17.7[10.2,30.6]$ times higher among people with top $1 \%$ erythrocyte counts $\left(p=1.1 \times 10^{-13}\right)$. While Loh et al. detected an interesting association, the authors only examined the extreme $1 \%$ of blood cell indices which resulted in a small sample size and accordingly wide confidence intervals. Their analysis was also a univariable statistical test which did not take into account potential confounding by other factors. A recently published study on 57, 987 men in Biobank Japan also reported associations among mLOY, increased thrombocyte and leukocyte counts ${ }^{64}$. While the authors did not find statistically significant negative associations between erythrocyte counts and continuous mLRR in univariable models, effect estimates indicate men with mLOY trended toward a decreased erythrocyte count. In the current study, we modeled mLOY in both continuous and categorical measures and employed multiple strategies to prevent potential confounding including multivariable models adjusting for potential risk factors as well as polygenic risk scores and a Mendelian randomization framework which are less susceptible to confounding.

A proposed biologic mechanism relating mLOY to disease risk has been through alteration of the immune system and its response to multiple factors ${ }^{3,65}$. mLOY could be associated with differences in blood counts either as a consequence of the mosaicism or as a response to one or more exposures that drive development and maintenance of mLOY. In particular, neutrophils have been suggested to promote tumor initiation by releasing reactive oxygen species, reactive nitrogen species, and proteases, as well as promote progression by activating senescent cancer cells and suppressing CD8+ T cell-mediated immune responses ${ }^{66}$. The role of thrombocytes is complex: classically platelet counts can be elevated in inflammatory conditions or select pediatric cancers (e.g., neuroblastoma). The literature also supports the association of elevated platelet counts with lung cancer risk ${ }^{59}$. Still it is plausible that thrombocytes could contribute to tumor progression by stimulating angiogenesis ${ }^{67}$ and activating thrombosis-related inflammation ${ }^{68}$. In addition, observational studies have found that the composition of blood cells including neutrophil-lymphocyte ratio and thrombocyte-lymphocyte ratio are associated with overall and disease-free survival in multiple cancers ${ }^{69}$.

Stratified analyses and inclusion of interaction terms in multivariable models demonstrated that smoking status interacts with mLOY and strengthened the association between mLOY and blood cell counts. Among the six blood cell types we reported, smoking status did not alter the directionality of associations between blood cell counts and mLOY except for lymphocytes. While mLOY was positively associated with lymphocyte counts in ever smokers, the association flipped in never smokers. This stark change in association along with a relatively mild association between mLOY and thrombocyte resulted in a larger effect size in association between TLR and mLOY in never smokers. Although high TLR has been suggested to be a prognostic biomarker reflecting systemic inflammation in cancer patients, the clinical utility of such a biomarker in healthy individuals remained uncertain. If deviations in blood cell counts were persistent over years or decades, these alterations could have an impact on immune regulation and immunosenscence, both likely contributors to chronic disease risk. Since we do not fully understand the underlying mechanisms, it is plausible that perturbations in genomic stability and cell cycle pathways could be altered as well. The strongest effect of mLOY is seen in the myeloid lineage, which is consistent with the immune hypothesis as a key element. The involvement of the myeloid lineage also suggests CHIP (a correlated phenotype) may have some relevance for these observed associations. We observed no relationship with basophil and eosinophil counts as well as overall lymphocyte counts.

It is notable that mLOY is associated with other non-leukocyte parameters, such as an increase in erythrocyte size and a decrease in hemoglobin concentration per cell as well as an increase in thrombocyte counts accompanies by a decrease in platelet distribution width. The changes in erythrocyte indices were similar to macrocytic hypochromic anemia. And the most common causes for macrocytic anemia were vitamin B12 and folate deficiency ${ }^{70}$. A report in 2016 on National Diet and Nutrition Surveys found that $12.4 \%$ and $6.4 \%$ women in childbearing age were deficient in serum vitamin B12 and folate despite $96 \%$ consumption of adequate B12 in $\mathrm{UK}^{71}$ suggesting that the current recommended intake of vitamin $\mathrm{B} 12$ and folate might require adjustment to accommodate difference in life style or genetic background which may influence bioavailability. The intake and deficiency of vitamin B12 and folate among men was under-studied. The cross-sectional design of current investigation is inadequate for elucidating causal relationship, but the potential involvement of mLOY and vitamin B12 and folate metabolism might warrant further study. The effect of mLOY on red blood cells is intriguing in light of the steady decline in hematopoietic regeneration after middle age- and indeed, our finding could be correlated but not causally related to $\operatorname{such}^{72}$.

Despite careful consideration of the effects of age and tobacco use in our analysis, we cannot rule out potential biases of residual confounding, or confounding by other unmeasured or unadjusted exposures. Increasing age and tobacco use are associated with both mLOY and changes in blood cell count indices and warrant careful consideration to remove potential confounding in our analysis of the association between mLOY and blood cell counts. To adjust for these effects, multivariable regression models adjusted for a 25-level smoking variable and allowed for non-linear relationships with age. Furthermore, stratified analyses restricted to strata of age group and smoking status found no major differences in overall analytical outcome except for in sub-analyses of lymphocytes; suggesting potential effect modification by age and tobacco use for lymphocytes. Additionally, we used a mLOY PRS as a genetic instrumental variable to further investigate the association between mLOY and blood cell counts. Again, blood cell indices that were significantly associated with $\mathrm{mLOY}$ in the observational data were also 
associated in this genetic analysis. The only exception was erythrocyte indices which remained highly significant but had effect sizes in the opposite direction, suggesting environmental contributors may have strong influence in this relationship.

Our analysis in the UK Biobank provides important evidence that mLOY in circulating blood cells is associated with changes in blood cell counts. Our cross-sectional investigation is unable to determine the temporality of this relationship (e.g., does mLOY precede changes in blood cell count). Results from our mediation analyses indicate mLOY could account for a small proportion of age and smoking-related effects on blood cell count; suggesting mLOY may precede changes in blood cell counts. Alternatively, the PRS analysis in women (who do not carry a Y chromosome) demonstrates a strong relationship between mLOY genetic susceptibility variants and blood cell counts independent of chromosome Y loss, suggesting blood cell count and mLOY may share common genetic risk factors related to genomic instability and cell cycle regulation or alternatively that genetic susceptibility to $\mathrm{mLOY}$ in men may be associated with genetic susceptibility to chromosomal alterations in women (e.g., mosaic chromosome X loss) that may have similar impacts on blood cell counts. Regardless, our analysis suggests mLOY and blood cell counts are highly associated and are relevant for underlying disease risk. Although the specific mechanisms responsible for the association between mLOY and blood cell counts are unclear, our work highlights the need to explore the functional bases of the reported associations. Future studies that examine the molecular impact of mLOY on cellular transcription, cell cycle regulation and differentiation are vital for expanding our understanding of how mLOY could have an impact on hematopoiesis, particularly in the aging population, and could provide novel insights into potential biological mechanisms responsible for the observed associations between $\mathrm{mLOY}$ and possible cancer and chronic disease risk.

Transparency statement. The lead author (the manuscript's guarantor) affirms that this manuscript is an honest, accurate, and transparent account of the study being reported; that no important aspects of the study have been omitted; and that any discrepancies from the study as originally planned (and, if relevant, registered) have been explained.

Patient and public involvement. The development of the research question or outcome measures was not informed by patients' priorities, experience, or preferences. No patients were involved in the design and conduct of the present study. There are no plans to disseminate the results to study participants.

\section{Data availability}

The data reported in this paper are available by application directly to the UK Biobank. The associations between $\mathrm{mLOY}$ and outcomes are provided in the supplementary data. Software code in R for the analyses is available upon request.

Received: 1 October 2019; Accepted: 4 February 2020;

Published online: 27 February 2020

\section{References}

1. Machiela, M. J. \& Chanock, S. J. Detectable clonal mosaicism in the human genome. Semin. Hematol. 50, 348-359 (2013).

2. Steensma, D. P. et al. Clonal hematopoiesis of indeterminate potential and its distinction from myelodysplastic syndromes. Blood 126, 9-16 (2015).

3. Forsberg, L. A. et al. Mosaic loss of chromosome $\mathrm{Y}$ in peripheral blood is associated with shorter survival and higher risk of cancer. Nat. Genet. 46, 624-628 (2014).

4. Zhou, W. et al. Mosaic loss of chromosome $Y$ is associated with common variation near TCL1A. Nat. Genet. 48, 563-568 (2016).

5. Loftfield, E. et al. Mosaic Y Loss Is Moderately Associated with Solid Tumor Risk. Cancer Res. 79, 461-466 (2019).

6. Zink, F. et al. Clonal hematopoiesis, with and without candidate driver mutations, is common in the elderly. Blood 130, 742-752 (2017).

7. Dumanski, J. P. et al. Mutagenesis. Smoking is associated with mosaic loss of chromosome Y. Science 347, 81-83 (2015).

8. Wong, J. Y. Y. et al. Outdoor air pollution and mosaic loss of chromosome Y in older men from the Cardiovascular Health Study. Environ. Int. 116, 239-247 (2018).

9. Dumanski, J. P. et al. Mosaic Loss of Chromosome Y in Blood Is Associated with Alzheimer Disease. Am. J. Hum. Genet. 98, 1208-1219 (2016).

10. Haitjema, S. et al. Loss of Y Chromosome in Blood Is Associated With Major Cardiovascular Events During Follow-Up in Men After Carotid Endarterectomy. Circ. Cardiovasc. Genet. 10, e001544 (2017).

11. Dumanski, J. P., Sundström, J. \& Forsberg, L. A. Loss of Chromosome Y in Leukocytes and Major Cardiovascular Events. Circ. Cardiovasc. Genet. 10, e001820 (2017).

12. Wright, D. J. et al. Genetic variants associated with mosaic Y chromosome loss highlight cell cycle genes and overlap with cancer susceptibility. Nat. Genet. 49, 674-679 (2017).

13. Loftfield, E. et al. Predictors of mosaic chromosome Y loss and associations with mortality in the UK Biobank. Sci. Rep. 8, 12316 (2018).

14. Thompson, D. J. et al. Genetic predisposition to mosaic Y chromosome loss in blood. Nature 1-6, https://doi.org/10.1038/s41586019-1765-3 (2019).

15. Sudlow, C. et al. UK biobank: an open access resource for identifying the causes of a wide range of complex diseases of middle and old age. PLoS Med. 12, e1001779 (2015).

16. Fry, A. et al. Comparison of Sociodemographic and Health-Related Characteristics of UK Biobank Participants With Those of the General Population. Am. J. Epidemiol. 186, 1026-1034 (2017).

17. Ishizaka, N. et al. Relationship between smoking, white blood cell count and metabolic syndrome in Japanese women. Diabetes Res. Clin. Pract. 78, 72-76 (2007).

18. Dixon, J. B. \& O’Brien, P. E. Obesity and the white blood cell count: changes with sustained weight loss. Obes. Surg. 16, 251-257 (2006).

19. Nakanishi, N. et al. Association of alcohol consumption with white blood cell count: a study of Japanese male office workers. J. Intern. Med. 253, 367-374 (2003).

20. Twig, G. et al. White blood cells count and incidence of type 2 diabetes in young men. Diabetes Care 36, 276-282 (2013). 
21. Karthikeyan, V. J. \& Lip, G. Y. H. White blood cell count and hypertension. J. Hum. Hypertens. 20, 310-312 (2006).

22. Lai, Y. C. et al. The association of plasma lipids with white blood cell counts: Results from the Multi-Ethnic Study of Atherosclerosis. J. Clin. Lipidol., https://doi.org/10.1016/j.jacl.2019.07.003 (2019).

23. Thompson, D. et al. Genetic predisposition to mosaic Y chromosome loss in blood is associated with genomic instability in other tissues and susceptibility to non-haematological cancers. bioRxiv 514026, https://doi.org/10.1101/514026 (2019).

24. Tingley, D., Yamamoto, T., Hirose, K., Keele, L. \& Imai, K. mediation: R Package for Causal Mediation Analysis. J. Stat. Softw. 59, 1-38 (2014).

25. Kamat, M. A. et al. PhenoScanner V2: an expanded tool for searching human genotype-phenotype associations. Bioinforma. Oxf. Engl. 35, 4851-4853 (2019).

26. Staley, J. R. et al. PhenoScanner: a database of human genotype-phenotype associations. Bioinforma. Oxf. Engl. 32, 3207-3209 (2016).

27. Crosslin, D. R. et al. Genetic variants associated with the white blood cell count in 13,923 subjects in the eMERGE Network. Hum. Genet. 131, 639-652 (2012).

28. Reiner, A. P. et al. Genome-wide association study of white blood cell count in 16,388 African Americans: the continental origins and genetic epidemiology network (COGENT). PLoS Genet. 7, e1002108 (2011).

29. Soranzo, N. et al. A genome-wide meta-analysis identifies 22 loci associated with eight hematological parameters in the HaemGen consortium. Nat. Genet. 41, 1182-1190 (2009).

30. Okada, Y. et al. Common variations in PSMD3-CSF3 and PLCB4 are associated with neutrophil count. Hum. Mol. Genet. 19, 2079-2085 (2010).

31. Ferreira, M. A. R. et al. Sequence variants in three loci influence monocyte counts and erythrocyte volume. Am. J. Hum. Genet. 85, 745-749 (2009).

32. Lo, K. S. et al. Genetic association analysis highlights new loci that modulate hematological trait variation in Caucasians and African Americans. Hum. Genet. 129, 307-317 (2011).

33. Li, J. et al. GWAS of blood cell traits identifies novel associated loci and epistatic interactions in Caucasian and African-American children. Hum. Mol. Genet. 22, 1457-1464 (2013).

34. Kamatani, Y. et al. Genome-wide association study of hematological and biochemical traits in a Japanese population. Nat. Genet. 42, $210-215(2010)$

35. Gudbjartsson, D. F. et al. Sequence variants affecting eosinophil numbers associate with asthma and myocardial infarction. Nat. Genet. 41, 342-347 (2009).

36. Astle, W. J. et al. The Allelic Landscape of Human Blood Cell Trait Variation and Links to Common Complex Disease. Cell 167, 1415-1429.e19 (2016).

37. Keller, M. F. et al. Trans-ethnic meta-analysis of white blood cell phenotypes. Hum. Mol. Genet. 23, 6944-6960 (2014).

38. Jain, D. et al. Genome-wide association of white blood cell counts in Hispanic/Latino Americans: the Hispanic Community Health Study/Study of Latinos. Hum. Mol. Genet. 26, 1193-1204 (2017).

39. Southam, L. et al. Whole genome sequencing and imputation in isolated populations identify genetic associations with medicallyrelevant complex traits. Nat. Commun. 8, 15606 (2017).

40. Yang, Q., Kathiresan, S., Lin, J.-P., Tofler, G. H. \& O’Donnell, C. J. Genome-wide association and linkage analyses of hemostatic factors and hematological phenotypes in the Framingham Heart Study. BMC Med. Genet. 8(Suppl 1), S12 (2007).

41. Kullo, I. J., Ding, K., Jouni, H., Smith, C. Y. \& Chute, C. G. A genome-wide association study of red blood cell traits using the electronic medical record. PloS One 5 (2010).

42. Ganesh, S. K. et al. Multiple loci influence erythrocyte phenotypes in the CHARGE Consortium. Nat. Genet. 41, 1191-1198 (2009).

43. van der Harst, P. et al. Seventy-five genetic loci influencing the human red blood cell. Nature 492, 369-375 (2012).

44. Gieger, C. et al. New gene functions in megakaryopoiesis and platelet formation. Nature 480, 201-208 (2011).

45. Ding, K. et al. Genetic Loci implicated in erythroid differentiation and cell cycle regulation are associated with red blood cell traits. Mayo Clin. Proc. 87, 461-474 (2012).

46. Uda, M. et al. Genome-wide association study shows BCL11A associated with persistent fetal hemoglobin and amelioration of the phenotype of beta-thalassemia. Proc. Natl. Acad. Sci. USA 105, 1620-1625 (2008).

47. van Rooij, F. J. A. et al. Genome-wide Trans-ethnic Meta-analysis Identifies Seven Genetic Loci Influencing Erythrocyte Traits and a Role for RBPMS in Erythropoiesis. Am. J. Hum. Genet. 100, 51-63 (2017).

48. Hodonsky, C. J. et al. Genome-wide association study of red blood cell traits in Hispanics/Latinos: The Hispanic Community Health Study/Study of Latinos. PLoS Genet. 13, e1006760 (2017).

49. Meisinger, C. et al. A genome-wide association study identifies three loci associated with mean platelet volume. Am. J. Hum. Genet. 84, 66-71 (2009).

50. Qayyum, R. et al. A meta-analysis and genome-wide association study of platelet count and mean platelet volume in african americans. PLoS Genet. 8, el002491 (2012).

51. Soranzo, N. et al. A novel variant on chromosome 7q22.3 associated with mean platelet volume, counts, and function. Blood 113, 3831-3837 (2009).

52. Ramsuran, V. et al. Duffy-null-associated low neutrophil counts influence HIV-1 susceptibility in high-risk South African black women. Clin. Infect. Dis. Off. Publ. Infect. Dis. Soc. Am. 52, 1248-1256 (2011).

53. Guerrero, J. A. et al. Novel loci involved in platelet function and platelet count identified by a genome-wide study performed in children. Haematologica 96, 1335-1343 (2011).

54. Schick, U. M. et al. Genome-wide Association Study of Platelet Count Identifies Ancestry-Specific Loci in Hispanic/Latino Americans. Am. J. Hum. Genet. 98, 229-242 (2016).

55. Lin, B. D. et al. 2SNP heritability and effects of genetic variants for neutrophil-to-lymphocyte and platelet-to-lymphocyte ratio. J. Hum. Genet. 62, 979-988 (2017).

56. Staley, O. Y. J. MendelianRandomization: Mendelian Randomization Package (2019).

57. Wickham, H. ggplot2: Elegant Graphics for Data Analysis. (Springer, 2016).

58. Lüdecke, D., Bartel, A., Schwemmer, C. \& Powell, C. sjPlot: Data Visualization for Statistics in Social Science (2019).

59. Zhu, Y. et al. Elevated platelet count appears to be causally associated with increased risk of lung cancer: A Mendelian randomization analysis. Cancer Epidemiol. Prev. Biomark. cebp.0356.2018, https://doi.org/10.1158/1055-9965.EPI-18-0356 (2019).

60. Roxburgh, C. S. D. \& McMillan, D. C. Role of systemic inflammatory response in predicting survival in patients with primary operable cancer. Future Oncol. Lond. Engl. 6, 149-163 (2010).

61. Dolan, R. D., McSorley, S. T., Horgan, P. G., Laird, B. \& McMillan, D. C. The role of the systemic inflammatory response in predicting outcomes in patients with advanced inoperable cancer: Systematic review and meta-analysis. Crit. Rev. Oncol. Hematol. 116, 134-146 (2017)

62. Salman, T. et al. Prognostic Value of the Pretreatment Neutrophil-to-Lymphocyte Ratio and Platelet-to-Lymphocyte Ratio for Patients with Neuroendocrine Tumors: An Izmir Oncology Group Study. Chemotherapy 61, 281-286 (2016).

63. Loh, P.-R. et al. Insights into clonal haematopoiesis from 8,342 mosaic chromosomal alterations. Nature 559, 350-355 (2018)

64. Terao, C. et al. GWAS of mosaic loss of chromosome Y highlights genetic effects on blood cell differentiation. Nat. Commun. 10, $1-10(2019)$.

65. Machiela, M. J. \& Chanock, S. J. The ageing genome, clonal mosaicism and chronic disease. Curr. Opin. Genet. Dev. 42, 8-13 (2017). 
66. Coffelt, S. B., Wellenstein, M. D. \& de Visser, K. E. Neutrophils in cancer: neutral no more. Nat. Rev. Cancer 16, 431-446 (2016).

67. Fuentes, E., Palomo, I. \& Rojas, A. Cross-talk between platelet and tumor microenvironment: Role of multiligand/RAGE axis in platelet activation. Blood Rev. 30, 213-221 (2016).

68. Olsson, A. K. \& Cedervall, J. The pro-inflammatory role of platelets in cancer. Platelets 29, 569-573 (2018).

69. Ethier, J.-L., Desautels, D., Templeton, A., Shah, P. S. \& Amir, E. Prognostic role of neutrophil-to-lymphocyte ratio in breast cancer: a systematic review and meta-analysis. Breast Cancer Res. BCR 19, 2 (2017).

70. Nagao, T. \& Hirokawa, M. Diagnosis and treatment of macrocytic anemias in adults. J. Gen. Fam. Med. 18, 200-204 (2017).

71. Sukumar, N., Adaikalakoteswari, A., Venkataraman, H., Maheswaran, H. \& Saravanan, P. Vitamin B12 status in women of childbearing age in the UK and its relationship with national nutrient intake guidelines: results from two National Diet and Nutrition Surveys. BMJ Open 6 (2016).

72. Mahlknecht, U. \& Kaiser, S. Age-related changes in peripheral blood counts in humans. Exp. Ther. Med. 1, 1019-1025 (2010).

73. Kratz, A., Ferraro, M., Sluss, P. M. \& Lewandrowski, K. B. Normal Reference Laboratory Values. N. Engl. J. Med. 351, 1548-1563 (2004).

74. Banfi, G. et al. Reticulocyte count, mean reticulocyte volume, immature reticulocyte fraction, and mean sphered cell volume in elite athletes: reference values and comparison with the general population. Clin. Chem. Lab. Med. 44, 616-622 (2006).

75. Budak, Y. U., Polat, M. \& Huysal, K. The use of platelet indices, plateletcrit, mean platelet volume and platelet distribution width in emergency non-traumatic abdominal surgery: a systematic review. Biochem. Medica 26, 178-193 (2016).

\section{Acknowledgements}

We thank Blanche P. Alter for her critical input on the manuscript. This work was supported by the Intramural Research Program of the National Cancer Institute. This research was conducted using the UK Biobank resource (application 21552). The UK Biobank was established by the Wellcome Trust, the Medical Research Council, the United Kingdom Department of Health, and the Scottish Government. The UK Biobank has also received funding from the Welsh Assembly Government, the British Heart Foundation, and Diabetes UK.

\section{Author contributions}

M.J.M. conceived and designed the study. E.L., N.D.F., S.J.C., M.J.M., M.Y. and W.Z. contributed to the acquisition and management of data. S.L., J.S. and M.J.M. contributed to the analysis of data. S.L., S.J.C. and M.J.M. drafted the first version of the manuscript. All authors contributed to the interpretation of the data, critically revised the manuscript and approved the final text.

\section{Competing interests}

The authors declare no competing interests.

\section{Additional information}

Supplementary information is available for this paper at https://doi.org/10.1038/s41598-020-59963-8.

Correspondence and requests for materials should be addressed to M.J.M.

Reprints and permissions information is available at www.nature.com/reprints.

Publisher's note Springer Nature remains neutral with regard to jurisdictional claims in published maps and institutional affiliations.

(c) Open Access This article is licensed under a Creative Commons Attribution 4.0 International

License, which permits use, sharing, adaptation, distribution and reproduction in any medium or format, as long as you give appropriate credit to the original author(s) and the source, provide a link to the Creative Commons license, and indicate if changes were made. The images or other third party material in this article are included in the article's Creative Commons license, unless indicated otherwise in a credit line to the material. If material is not included in the article's Creative Commons license and your intended use is not permitted by statutory regulation or exceeds the permitted use, you will need to obtain permission directly from the copyright holder. To view a copy of this license, visit http://creativecommons.org/licenses/by/4.0/.

(c) The Author(s) 2020 